\title{
Medication discrepancies and associated risk factors identified among elderly patients discharged from a tertiary hospital in Singapore
}

Farooq $\underline{\mathrm{Akram}}^{1,2}$, FAMS, MRCP, Paul J $\underline{\mathrm{Huggan}}^{3}$, FRACP, Valencia $\underline{\mathrm{Lim}}^{2}$, MBBS, Yufang $\underline{\mathrm{Huang}}{ }^{2}$, MBBS, Fahad Javaid Siddiqui ${ }^{4,5}$, MSc, Pryseley Nkouibert $\underline{\text { Assam }}^{4,5}$, PhD, Reshma A Merchant ${ }^{1,2}$, FAMS, MRCP

\begin{abstract}
INTRODUCTION Medication discrepancies and poor documentation of medication changes (e.g. lack of justification for medication change) in physician discharge summaries can lead to preventable medication errors and adverse outcomes. This study aimed to identify and characterise discrepancies between preadmission and discharge medication lists, to identify associated risk factors, and in cases of intentional medication discrepancies, to determine the adequacy of the physician discharge summaries in documenting reasons for the changes.

METHODS A retrospective clinical record review of 150 consecutive elderly patients was done to estimate the number of medication discrepancies between preadmission and discharge medication lists. The two lists were compared for discrepancies (addition, omission or duplication of medications, and/or a change in dosage, frequency or formulation of medication). The patients' clinical records and physician discharge summaries were reviewed to determine whether the discrepancies found were intentional or unintentional. Physician discharge summaries were reviewed to determine if the physicians endorsed and documented reasons for all intentional medication changes.

RESULTS A total of 279 medication discrepancies were identified, of which 42 were unintentional medication discrepancies (35 were related to omission/addition of a medication and seven were related to a change in medication dosage/ frequency) and 237 were documented intentional discrepancies. Omission of the baseline medication was the most common unintentional discrepancy. No reasons were provided in the physician discharge summaries for 54 (22.8\%) of the intentional discrepancies.

CONCLUSION Unintentional medication discrepancies are a common occurrence at hospital discharge. Physician discharge summaries often do not have adequate information on the reasons for medication changes.
\end{abstract}

Keywords: documentation of reasons, medication discrepancies, physician discharge summaries

\section{INTRODUCTION}

Medication discrepancies are defined as inconsistencies between two or more medication lists; they commonly occur among geriatric patients at the time of hospital discharge. ${ }^{(1)}$ Discrepancies can lead to preventable medication errors and adverse events. ${ }^{(2-5)}$ In a recent study by Coleman et al, $14.1 \%$ of elderly patients experienced more than one medication discrepancy post-hospitalisation. ${ }^{(6)}$ Other studies found that $23 \%-49 \%$ of hospitalised patients experience more than one medical error following discharge from a teaching hospital, $72 \%$ of which are related to medications. ${ }^{(3,7,8)}$

Unintentional medication discrepancies, which often result from unintended medication changes, pose a risk to patient safety. Even intentional discrepancies, if poorly communicated and inadequately documented in physician discharge summaries, can leave the next care providers with unanswered questions. Preventable errors can then occur, which may affect patient safety, especially during early post-discharge. ${ }^{(9)}$ Notably, it has been reported that information regarding discharge medications are absent from $2 \%-40 \%$ of discharge summaries. ${ }^{(10,11)}$

One way to reduce medication discrepancy risk and promote patient safety is to have medication reconciliation at all interfaces of care. ${ }^{(1)}$ This was a designated national patient safety goal in 2005 by The Joint Commission, the United States. ${ }^{(12,13)}$ In order for reconciliation efforts to be effective, institutions need a more thorough understanding of the nature of the problem that reconciliation aims to prevent. ${ }^{(14)}$ The present study was designed to identify and characterise the discrepancies between preadmission and discharge medication lists, in order to define the associated risk factors, and to determine whether physicians document and provide reasons for the medication changes in discharge summaries. To the best of our knowledge, this is the first study in Singapore to address this patient safety issue in the setting of an acute tertiary hospital.

\section{METHODS}

Approval to carry out a retrospective clinical record review of 150 patients was obtained from the National Healthcare Group, Domain Specific Review Board, Singapore. A total of 229 consecutive patients aged $\geq 65$ years and admitted to any of the two Internal Medicine wards in National University Hospital, Singapore, between 25 May 2011 and 28 June 2011 were screened. Of these 229 patients, only 150 fulfilled the inclusion criteria. To be eligible for this study, patients had to have a 
traceable prescription in the National Healthcare Group's Cluster Patients Record System (CPRS), with evidence of a prescription refill for a pre-specified set of 21 chronic diseases. These 21 chronic diseases were identified in a previous study at the same institution (unpublished data) and the patients were on treatment for them at the time of admission (Table I). Patients were excluded if they were transferred to other wards, taken over by other subspecialties or expired before hospital discharge.

The following patient data was collected: age, gender, number of comorbidities, functional status, number of preadmission and discharge medications, length of stay, number of past hospitalisations, documentation of dedicated clinical rounds carried out on the day before discharge or on the day of discharge (early morning), and whether discharge was carried out on a weekend or a public holiday. All non-electronic preadmission medication prescriptions, which are usually obtained from private general practitioners (GPs), were excluded because information related to such prescriptions was not consistently available in the clinical records. The lists of preadmission and discharge medications were obtained from the CPRS and physician discharge summaries. Computerised provider order entry and pharmacy records are typically documented and available in the CPRS. Medications obtained from private GPs and over-thecounter medications were manually entered by clinicians, as such information is not automatically populated or updated.

In our institution, the general medicine clinical pharmacists caring for patients routinely perform admission and discharge medication reconciliation. Our electronic system has features that facilitate electronic collection and transfer of detailed medication information obtained through medication reconciliation (from the time of admission to discharge). This detailed medical information is routinely available to the discharging teams for the preparation of discharge prescriptions.

Preadmission and discharge medication lists were compared for any discrepancy (e.g. addition, omission, duplication and/or changes in dose, frequency or formulation of medication). Patient clinical records and physician discharge summaries were reviewed to determine if the discrepancies found were intentional or unintentional. A discrepancy was defined as an inconsistency between the two medication lists of a patient, regarding the presence, absence, dosage, route, frequency or formulation of a medication. An unintentional discrepancy was defined as one that lacked any documentation of medication change in either the clinical record or the physician discharge summary. An intentional discrepancy was defined as one associated with clear documentation of the intended change in the clinical record and/or the physician discharge summary. An intentional discrepancy was considered conflicting if the discharge medication was found to be different in any way from the physician's orders in the clinical order or discharge summary. A discrepancy was deemed inconclusive if there were limitations to the determination of the physician's intent (e.g. illegible or faded handwritten inpatient case notes). Finally, we documented whether intentional discrepancies were communicated in the discharge summaries.
Table I. The $\mathbf{2 1}$ chronic diseases used in the present study to define preadmission medications.

1. Ischaemic heart disease

2. Diabetes mellitus

3. Hypertension

4. Stroke

5. Dysrhythmia

6. Hyperlipidaemia

7. Congestive cardiac failure

8. Asthma

9. Chronic obstructive pulmonary disease

10. Benign prostatic hyperplasia

11. Hypothyroidism

12. Hyperthyroidism

13. Epilepsy

14. Depression

15. Psychosis

16. Parkinson's disease

17. Dementia

18. Rheumatoid arthritis

19. Gout

20. Chronic kidney disease

21. Osteoporosis

Discrepancies in the preadmission and discharge medication lists were assessed by noting the overall presence of differences between the two lists and enumerating the type of discrepancies between the two lists. Assignment of reasons for the observed discrepancies was also assessed. 95\% confidence intervals (Cls) were calculated using the normal approximation method. ${ }^{(15,16)}$ The association between the various risk factors and the presence of unintentional or conflicting discrepancy (UCD) was characterised using odds ratios (ORs) and their respective 95\% Cls. Univariate and multivariate logistic regression models were used to estimate the unadjusted and adjusted ORs between the risk factors and the incidence of UCDs. ${ }^{(15,16)}$

Due to the exploratory nature of this study, a rigorous model-building approach was adopted. First, univariate logistic regression models were employed to assess the association between each of the potential risk factors (i.e. age, gender, number of preadmission and discharge medications, number of comorbidities and functional status as reflected by the Barthel index) and the incidence of UCDs. Second, stepwise, forward and backward automated variable selection methods were used to identify the independent risk factors for UCDs. ${ }^{(17-19)}$ The significance level for a risk factor to enter or stay in the model was set at 0.20 . Third, variables that were selected by any of the automated variable selection methods were included in a multivariate logistic regression model, and a backward elimination procedure was performed by the statistician based on a significance level of 0.05 . The effect of sensible interactions between independent risk factors was also evaluated. The Hosmer-Lemeshow test was used to evaluate the goodness of fit of the multivariate logistic regression model. ${ }^{(20)}$ The significance level, which was adjusted for multiple testing, was set at $5 \%$ unless 
stated otherwise. Analysis was performed using the Statistical Analysis System version 9.2 (SAS Institute Inc, Cary, NC, USA).

\section{RESULTS}

The characteristics of the study population are shown in Table II. Among the 150 consecutive patients in the study cohort, $89(59.3 \%)$ were female and $61(40.7 \%)$ were male. They had a median age (interquartile range [IQR]) of $78(72-83)$ years. The mean (IQR) number of preadmission medications was 5 (3-7) and the mean (IQR) number of baseline comorbidities was $4(2-5)$. Among the patients, $18.0 \%$ presented with severe baseline functional dependency (i.e. Barthel index score $\leq 4$ ). The median (IQR) length of stay was 5 (4-9) days.

For the 760 preadmission medications in our cohort, 42 unintentional medication discrepancies (35 were related to omission or addition of a medication and seven were related to a change in dosage and frequency) were found in 35 (23.3\%) patients. Omission of the baseline medication was the most common unintentional discrepancy. 237 documented intentional discrepancies were found in 115 (76.7\%) patients. Among the 237 documented intentional discrepancies, 5 (2.1\%) were intentional with conflict. No reasons were assigned in the discharge summaries for $54(22.8 \%)$ of the intentional discrepancies (Fig. 1). Dedicated 'day before discharge' and 'day of discharge early morning' clinical rounds were documented for $90.0 \%$ and $97.3 \%$ of the study patients, respectively; 27 (18.0\%) patients were discharged on a weekend or public holiday (Table II).

The incidence of UCDs was not significantly related to the patients' age, number of preadmission and discharge medications, number of comorbidities or baseline functional status. However, the incidence of UCDs was significantly related to the number of discrepancies per patient on discharge (OR 1.29, 95\% Cl 1.06-1.56, $p=0.009$ ), indicating that patients with a higher number of medication changes during admission are more likely to have a UCD (Table III).

\section{DISCUSSION}

The present study is one of the few studies that examine the prevalence of medication discrepancies among elderly patients discharged from a tertiary care hospital; to the best of our knowledge, it is the first such study conducted in Singapore. Among the hospitalised elderly in our study, we found that $23.3 \%$ had at least one actual unintentional medication discrepancy on discharge. This value is much higher than that (14.1\%) reported by Coleman et al in their prospective study. ${ }^{(6)}$ In that study, the medication discrepancies were identified in the post-discharge setting. Several other studies have reported higher incidences of medication discrepancies (between $41 \%$ and $56 \%$ ); ${ }^{(4,22-24)}$ these differences in the incidence of medication discrepancies are likely due to the different study designs and study settings.

Omission and addition of a new medication were the most common actual unintentional discrepancies identified in the present study. This is consistent with the findings of other studies. ${ }^{(4,22,24)}$ Although intentional discrepancies with conflict occurred less frequently than unintentional discrepancies in our
Table II. Characteristics of the study population $(n=150)$.

\begin{tabular}{|c|c|}
\hline Characteristic & No. (\%) \\
\hline Female gender & $89(59.3)$ \\
\hline Age (yr)* & 78 (72-83); (65-100) \\
\hline No. of comorbidities* & $4(2-5) ;(0-8)$ \\
\hline No. of preadmission medications* & $5(3-7) ;(0-15)$ \\
\hline No. of hospitalisations in last $1 \mathbf{y r}^{*}$ & $1(0-2) ;(0-6)$ \\
\hline No. of discharge medications* & $5(3-7) ;(0-15)$ \\
\hline Length of stay (day)* & $5(4-9) ;(1-40)$ \\
\hline \multicolumn{2}{|l|}{$\begin{array}{l}\text { Barthel index }{ }^{+} \text {score ( } 2 \text { wk before the } \\
\text { acute event) }\end{array}$} \\
\hline High ( $\geq 12)$ & $102(68.0)$ \\
\hline Intermediate (9-11) & $15(9.9)$ \\
\hline Low $(5-8)$ & $6(4.0)$ \\
\hline Very low $(\leq 4)$ & $27(18.0)$ \\
\hline \multicolumn{2}{|l|}{ Barthel index ${ }^{+}$score (at admission) } \\
\hline High ( $\geq 12$ ) & $84(56.0)$ \\
\hline Intermediate (9-11) & $19(12.7)$ \\
\hline Low $(5-8)$ & $14(9.3)$ \\
\hline Very low $(\leq 4)$ & $33(22.0)$ \\
\hline Received 'day before discharge' rounds & $135(90.0)$ \\
\hline $\begin{array}{l}\text { Received 'day of discharge' early } \\
\text { morning rounds }\end{array}$ & $146(97.3)$ \\
\hline $\begin{array}{l}\text { Discharged on a weekend or public } \\
\text { holiday }\end{array}$ & $27(18.0)$ \\
\hline
\end{tabular}

*Data presented as median (interquartile range); (range). †10-item, 20-point Barthel index by Collin et al. ${ }^{(21)}$

study, they are equally undesirable and represent medication error. Such discrepancies are significant as they may confuse both the patients and their community clinicians. Nickerson et al found that $90.9 \%$ of discrepancies have a potentially significant or very significant clinical impact, ${ }^{(24)}$ while other studies have shown that medication discrepancies are associated with increased rates of rehospitalisation $^{(25)}$ and increased healthcare-associated costs. ${ }^{(26)}$ Although we did not assess the risk of possible negative outcomes caused by such discrepancies, the implications and risk of adverse events can be inferred.

In contrast to previous studies, ${ }^{(1,6,27)}$ we did not find any associations between number of unintentional medication discrepancies and patient age, number of comorbidities present and number of medications at admission. The absence of these associations may be due to the small sample size, the different patient population or the different study setting. It may have also been due to our unit's robust system of dedicated clinical rounds, which are carried out on the day before discharge or in the early morning of the day of discharge. These clinical rounds may have resulted in a lower overall incidence of unintentional medication discrepancy among patients with complex medical problems.

One of the objectives of the present study was to audit the adequacy of the physician discharge summaries and to identify gaps in the current discharge process, for the purpose of enabling targeted improvement. In the present study, 29 of the patients with intentional medication changes were discharged without justification given in the physician discharge summaries. It 


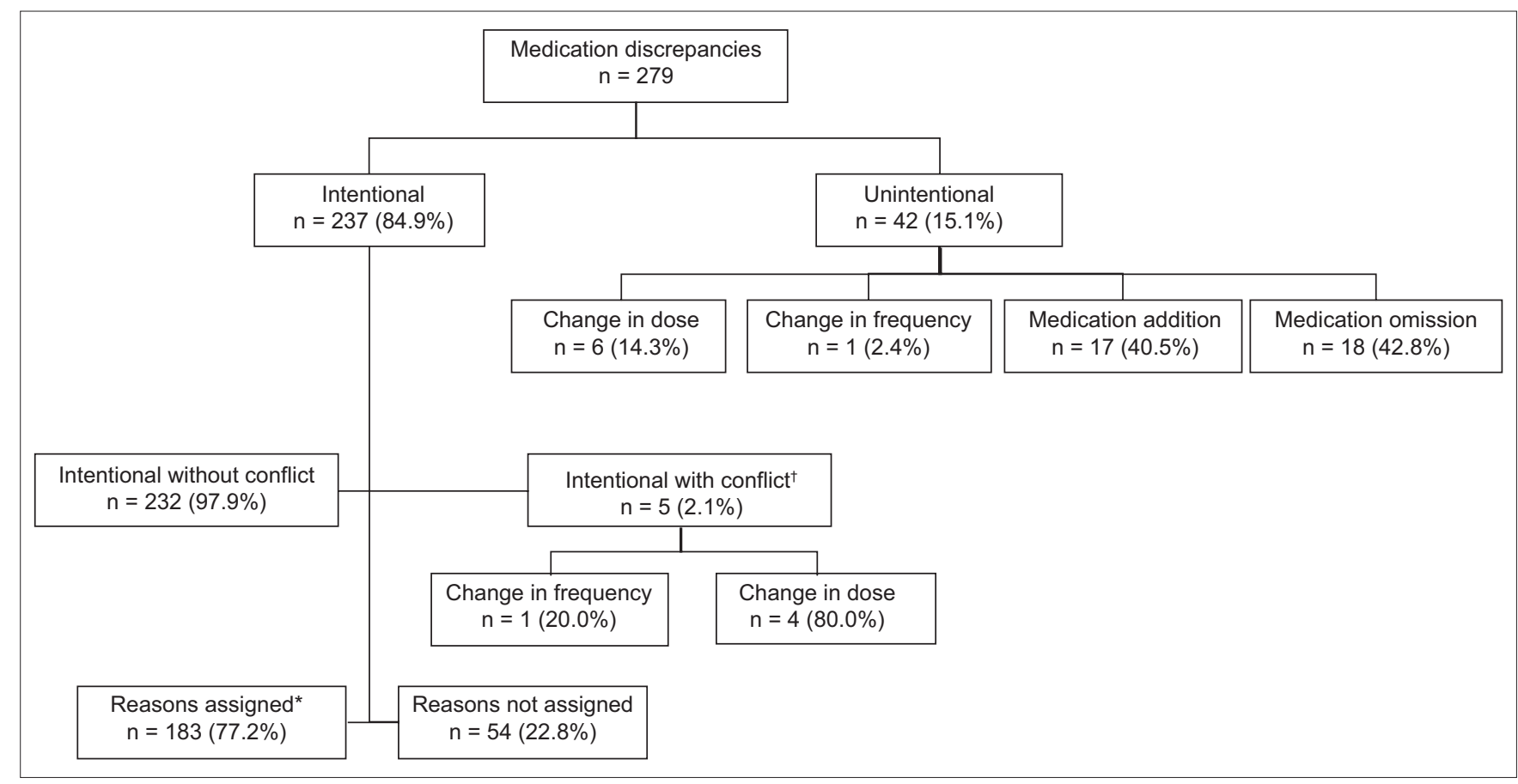

Fig. 1. Chart shows the characteristics and types of medication discrepancies. *Assignment of reasons for intentional medication discrepancies in physician discharge summaries is considered the best medical practice. tDiscrepancies (both intentional and unintentional) with conflict represent medication error and are considered undesirable.

Table III. Binary logistic regression analysis of patient-related risk factors for unintentional medication discrepancies at hospital discharge.

\begin{tabular}{lcc}
\hline Characteristic & OR (95\% CI) & p-value \\
\hline Age (yr) & $1.00(0.95-1.05)$ & 0.961 \\
Gender & $1.55(0.69-3.48)$ & 0.286 \\
No. of comorbidities & $0.92(0.74-1.15)$ & 0.473 \\
No. of preadmission medications & $1.09(0.95-1.25)$ & 0.243 \\
No. of discharge medications & $1.10(0.96-1.26)$ & 0.169 \\
No. of hospitalisation in the & $0.93(0.71-1.21)$ & 0.572 \\
past 1 yr & $1.03(0.97-1.09)$ & 0.298 \\
Length of stay & $1.08(0.77-1.51)$ & 0.659 \\
Barthel index (2 wk before the & $1.02(0.97-1.08)$ & 0.467 \\
acute event) & & \\
Barthel score (2 wk before the & & 0.576 \\
acute event) & $0.98(0.93-1.04)$ & 0.009 \\
Barthel score (on admission) & $1.29(1.06-1.56)$ & \\
No. of discrepancies per patient & & \\
on discharge & & \\
\hline
\end{tabular}

$\mathrm{Cl}$ : confidence interval; OR: odds ratio

was logical that no patient with an unintentional medication discrepancy had justification documented for the discrepancy. If any discrepancy in the preadmission medications was noted, the pharmacist overseeing the discharge prescriptions would call the discharging physician to enquire whether the change was intentional. Despite this measure and the dedicated discharge rounds, one out of four patients in the present study was discharged with at least one unintentional medication discrepancy.

The findings of the present study provide important insights into the performance of the current medication reconciliation process and its inadequacies. Firstly, medication reconciliation was done mainly by acquiring preadmission medication lists from electronic medication records, which are often inaccurate. ${ }^{(28)}$ A recent study, which was conducted in a wellestablished healthcare system that has broadly integrated electronic health records, showed that $60 \%$ of the patients had one or more medication discrepancies. ${ }^{(28)}$ Electronic health records are currently not widely accessible in Singapore; the records are not linked to community pharmacies and private healthcare providers, which constitute the bulk of primary healthcare in Singapore. ${ }^{(29)}$ Secondly, we did not find any documented evidence to suggest that a comprehensive medication history, which includes multiple sources of information, was obtained in all patients to generate an accurate preadmission medication list. Thirdly, a recent study conducted in the Netherlands showed that insufficient knowledge among healthcare professionals, unclear task allocations and lack of collaboration among the multidisciplinary stakeholders were important barriers to successful implementation of medication reconciliation. ${ }^{(30)}$ Even though we could not assess these factors in the present study due to its retrospective nature, concerns can be raised regarding adequacy of communication and collaboration among members of the multidisciplinary team, in view of the significant number of unintentional discrepancies. Finally, we noted a lack of documentation regarding medication changes in the physician discharge summaries. These summaries, which serve as the primary document for communicating a patient's care plan to post-hospital healthcare providers, may leave the next healthcare provider with limited information regarding medications that were stopped and reasons for their discontinuation, and reasons for the introduction of new medications (if any). This finding points 
toward a system of care that places the burden of transcription and clinical documentation on a limited number of clinical staff who face competing responsibilities. Residents are particularly vulnerable to lapses that may result from time demands, workload and exhaustion. ${ }^{(31)}$

The findings of the present study should be interpreted in light of several limitations. The review of the two medication lists of each patient was carried out retrospectively; in other words, the investigators were not able to speak with the physician, the patient or their family members (which would have been possible if the study was done prospectively). In addition, the review of the medications prescribed was limited to the 21 pre-specified chronic medical conditions. The medication prescription lists were obtained from an electronic patient record system and only medications with a refill supply that was sufficient to last till the day of index admission were included. This step was taken to ensure that the list obtained was the most accurate preadmission medication list. However, this may have resulted in a relatively lower overall incidence of unintentional discrepancy, as many medications were excluded from review. Furthermore, we did not review the outpatient clinic notes. These notes may contain information about dose adjustments or changes in medications. It is possible that some patients had dose adjustments or changes in medications, such as drug omission, during their visits to hospital clinics. These changes would not have been reflected in the electronic medication records as the changes may not have required new prescriptions.

The findings of the present study support the need for a comprehensive, structured and multidisciplinary team approach to ensure the best possible practice of medication reconciliation at all transitions of care. The High 5s, a medication reconciliation standard operating procedure, ${ }^{(32)}$ is one of the five patient safety protocols developed by the Institute for Safe Medication Practice, Canada. It consists of a standardised, two-step process. The first step outlines the acquisition of a 'best possible medication history' that is more comprehensive than a routine primary medication history, which is often a quick preliminary medication history that may not include multiple sources of information. The second step deals with the resolution of inaccuracies in the current medication history (i.e. medication discrepancies) in consultation with the principal practitioner. It should lead to the development of a 'best possible discharge medication list', which should then be compared against discharge prescriptions. Kwan et al showed that the number of patients with inaccuracies in their current medication history can be halved with the introduction of this intervention. ${ }^{(33)}$

It is essential to recognise that effective communication and partnership among hospital staff, community healthcare providers and patients is the key to a successful medication reconciliation process. Patient empowerment through medication education, which ensures that the patient comprehends the medication instructions, should be an endpoint of discharge counselling; the endpoint should not just be the act of delivering the information. Moving forward, there should be plans to develop a single and updated system of reconciled medication lists and care plans that is electronically and manually accessible to patients, their caregivers, physicians, alternative care practitioners, clinics, hospitals, home health aides, nursing homes and other people who participate in the care of a patient. The development of such a system would be an important milestone in medication safety.

To conclude, we found that medication discrepancies are a common occurrence in elderly patients at the time of discharge in our hospital. Physician discharge summaries did not adequately document and justify medication changes. We support and recommend the trial and implementation of the 'best possible medication reconciliation' practice model and other effective strategies, for the purpose of improving the quality of discharge communication in relation to patient medication.

\section{REFERENCES}

1. Stitt DM, Elliott DP, Thompson SN. Medication discrepancies identified at time of hospital discharge in a geriatric population. Am J Geriatr Pharmacother 2011; 9:234-40.

2. Cornish PL, Knowles SR, Marchesano R, et al. Unintended medication discrepancies at the time of hospital admission. Arch Intern Med 2005; 165:424-9.

3. Forster AJ, Murff HJ, Peterson JG, Gandhi TK, Bates DW. The incidence and severity of adverse events affecting patients after discharge from the hospital. Ann Intern Med 2003; 138:161-7.

4. Vira T, Colquhoun M, Etchells E. Reconcilable differences: correcting medication errors at hospital admission and discharge. Qual Saf Health Care 2006; 15:122-6.

5. Kripalani S, Jackson AT, Schnipper JL, Coleman EA. Promoting effective transitions of care at hospital discharge: a review of key issues for hospitalists. J Hosp Med 2007; 2:314-23.

6. Coleman EA, Smith JD, Raha D, Min SJ. Posthospital medication discrepancies: prevalence and contributing factors. Arch Intern Med 2005; 165:1842-7.

7. Moore C, Wisnivesky J, Williams S, McGinn T. Medical errors related to discontinuity of care from an inpatient to an outpatient setting. J Gen Intern Med 2003; 18:646-51

8. Forster AJ, Clark HD, Menard A, et al. Adverse events among medical patients after discharge from hospital. CMAJ 2004; 170:345-9.

9. Cua YM, Kripalani S. Medication use in the transition from hospital to home. Ann Acad Med Singapore 2008; 37:136-6.

10. Kripalani S, LeFevre F, Phillips CO, et al. Deficits in communication and information transfer between hospital-based and primary care physicians: implications for patient safety and continuity of care. JAMA 2007; 297:831-41.

11. van Walraven C, Weinberg AL. Quality assessment of a discharge summary system. CMAJ 1995; 152:1437-42.

12. Forster AJ, Murff HJ, Peterson JF, Gandhi TK, Bates DW. Adverse drug events occurring following hospital discharge. J Gen Intern Med 2005; 20:317-23.

13. The Joint Commission. Ambulatory Health Care National Patient Safety Goals 2015. Available at: http://www.jointcommission.org/ assets/1/6/2015_NPSG_AHC1.PDF. Accessed January 5, 2015.

14. Pippins JR, Gandhi TK, Hamann C, et al. Classifying and predicting errors of inpatient medication reconciliation. J Gen Intern Med 2008; 23:1414-22.

15. Agresti A. Categorical Data Analysis. 2nd ed. New York: John Wiley \& Sons, 2002.

16. Agresti A. An Introduction to Categorical Data Analysis. 2nd ed. New York: John Wiley \& Sons, 2007.

17. Hocking RR. A biometrics invited paper. The analysis and selection of variables in linear regression. Biometrics 1976; 32:1-49.

18. Miller AJ. Selection of subsets of regression variables. J R Stat Soc Series A $1984 ; 147: 389-425$.

19. Miller A. Subset Selection in Regression. 2nd ed. Boca Raton, FL: Chapman \& Hall/CRC, 2002

20. Hosmer DW, Lemeshow S. Applied Logistic Regression. New York: John Wiley \& Sons, 2004.

21. Collin C, Wade DT, Davies S, Horne V. The Barthel ADL Index: a reliability study. Int Disabil Stud. 1988; 10:61-3.

22. Bolas H, Brookes K, Scott M, McElnay J. Evaluation of a hospital-based 
community liaison pharmacy service in Northern Ireland. Pharm World Sci $2004 ; 26: 114-20$.

23. Whittington J, Cohen H. OSF healthcare's journey in patient safety. Qual Manag Health Care 2004; 13:53-9.

24. Nickerson A, MacKinnon NJ, Roberts N, Saulnier L. Drug-therapy problems, inconsistencies and omissions identified during a medication reconciliation and seamless care service. Healthc Q 2006; 8:65-72.

25. Walker PC, Berstein SJ, Jones JN, et al. Impact of a pharmacist-facilitated hospital discharge program: a quasi-experimental study. Arch Intern Med 2009; 169:2003-10.

26. Dedhia $P$, Kravet $S$, Bluger J, et al. A quality improvement intervention to facilitate the transition of older adults from three hospitals back to their homes. J Am Geriatr Soc 2009; 57:1540-6.

27. Unroe KT, Pfeiffenberger T, Riegelhaupt S, et al. Inpatient medication reconciliation at admission and discharge: A retrospective cohort study of age and other risk factors or medication discrepancies. Am J Geriatr Pharmacother 2010; 8:115-26.

28. Linsky A, Simon SR. Medication discrepancies in integrated electronic health records. BMJ Qual Saf 2013; 22:103-9.

29. Lee KH. The hospitalist movement--a complex adaptive response to fragmentation of care in hospitals. Ann Acad Med Singapore 2008; 37:145-50.

30. van Sluisveld N, Zegers M, Natsch S, Wollersheim H. Medication reconciliation at hospital admission and discharge: insufficient knowledge, unclear task reallocation and lack of collaboration as major barriers to medication safety. BMC Health Serv Res 2012; 12:170.

31. Haney EM, Nicolaidis C, Hunter A, et al. Relationship between resident workload and self-perceived learning on inpatient medicine wards: a longitudinal study. BMC Med Educ 2006; 6:35.

32. World Health Organization. Assuring medication accuracy at transitions in care: medication reconciliation. High 5's project: Action on patient safety Standard Protocol. Available at: http://www.who.int/patientsafety/ implementationsolution/high5s-sop.pdf. Accessed January 1, 2014

33. Kwan Y, Fernandes OA, Nagge JJ, et al. Pharmacist medication assessments in a surgical preadmission clinic. Arch Intern Med 2007; 167:1034-40. 